\title{
INTRAVENOUS REGIONAL ANESTHESIA VERSUS AXILLARY NERVE BLOCK FOR AMBULATORY HAND SURGERY: \\ A PROSPECTIVE, RANDOMIZED TRIAL \\ Stan Outtier MD ${ }^{1}$, Steffen Rex MD PhD ${ }^{1}$, Marc Van De Velde MD PhD EDRA ${ }^{1}$, An Teunkens MD ${ }^{1}$ \\ ${ }^{1}$ Department of Anesthesiology, University Hospitals Leuven, Belgium
}

\section{Background \& Aims}

Regional anesthesia is a popular alternative for general anesthesia in ambulatory hand surgery, resulting in faster recovery and, discharge times. Two commonly applied techniques are Intravenous Regional Anesthesia (IVRA) and the Axillary Block (AB). Traditionally, analgesic efficacy, adverse events and safety are studied to compare regional anesthesia techniques. In our study, we aim to evaluate patient satisfaction with both techniques.

\section{Methods}

After obtaining ethical committee approval and written informed consent, 120 patients undergoing hand surgery were included in this prospective trial and randomized into the IVRA-group and the AB-group. Patient satisfaction as the primary outcome was assessed with the EVAN-LR questionnaire. Secondary outcomes included induction time, tourniquet time, discharge time and length of hospital stay, block quality, pain scores, need for supplemental analgesia and conversion to general anesthesia (GA).

\section{Results}

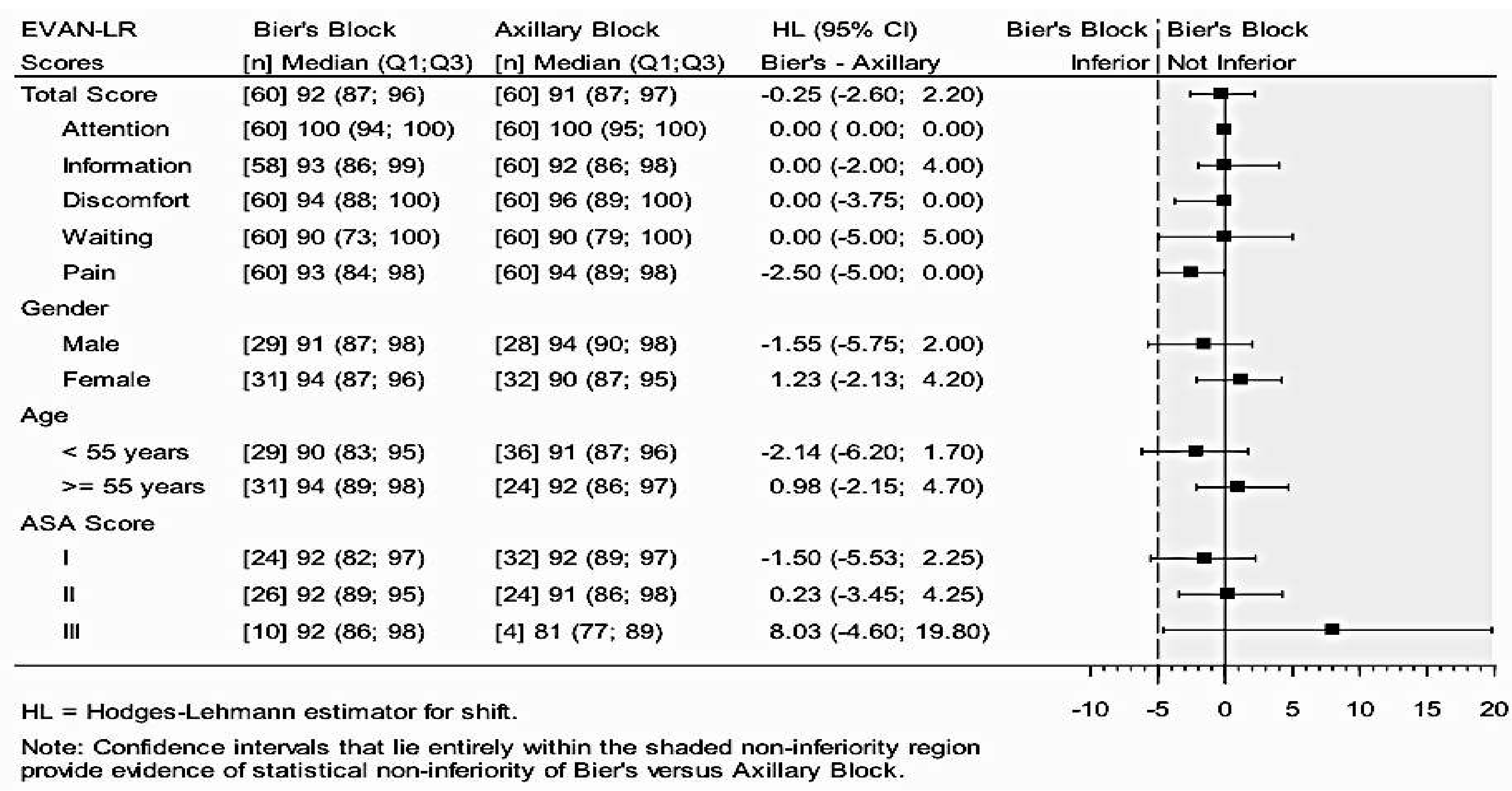

There was no difference in patient satisfaction between the IVRA-group and the AB-group (table_1). Induction time, hospital stay and discharge time were significant longer in AB-group $(p<0.001)$. Intra- and postoperative pain scores were significantly higher and tourniquet time was longer in the IVRA-group $(p<0.001)$, but no difference in need for supplemental analgesia or conversion to GA was shown. No differences were found in block quality, nor in pain scores and need for supplemental analgesia after discharge.

\section{Conclusion}

Patients undergoing IVRA were equally satisfied as patients with $A B$, and had a shorter induction time, discharge time and hospital stay. However, higher pain scores with IVRA suggest the need for a more appropriate pain management for these patients. 